\title{
PROPOSED BEAM DIAGNOSTICS INSTRUMENTATION FOR THE LANSCE REFURBISHMENT PROJECT *
}

\author{
J. D. Gilpatrick ${ }^{\#}$, B. Blind, M. Borden, J. Erickson, R. McCrady, M. S. Gulley, S. Kurennoy, J. \\ O'Hara, M. Oothoudt, C. Pillai, J. Power, L. Rybarcyk, F. Shelley, LANL, Los Alamos, NM, \\ 87545, U.S.A.
}

\begin{abstract}
Presently, the Los Alamos National Laboratory is in the process of planning a refurbishment of various subsystems within its Los Alamos Neutron Science Center accelerator facility. A part of this LANSCE facility refurbishment will include some replacement of and improvement to existing older beam-diagnostics instrumentation. While plans are still being discussed, some instrumentation that is under improvement or replacement consideration are beam phase and position measurements within the $805-\mathrm{MHz}$ side-coupled cavity linac, slow wire profile measurements, typically known as wire scanners, and possibly additional installation of fast ionization-chamber loss monitors. This paper will briefly describe the requirements for these beam measurements, what we have done thus far to answer these requirements, and some of the technical issues related to the implementation of the instrumentation.
\end{abstract}

\section{BEAM INSTRUMENTATION IMPROVEMENTS}

The LANSCE-R project is expected to improve or enhance the operation of some of the older sub-systems within the LANCE facility. From a beam-diagnostic instrumentation perspective, this includes those devices that have either become sufficiently old to have parts that are in some cases no longer available or have become devices whose maintenance has become of primary concern. The two leading contenders for this improvement are the Beam Position and Phase Monitors (BPPM) within the $805-\mathrm{MHz}$ linac and the wire scanners throughout the facility [1]. These beam-position and -phase measurement systems will provide additional noninterceptive information of the beam's central position and phase within the linac during tuning and operation. These additional measurements will provide the tuners of the linac new instrumentation to help reduce the time it takes to bring the linac and facility to an operational state. Other systems, such as the slow wire scanner profile measurements, used throughout the linac, have one of the highest maintenance costs due to either old or antiquated equipment or sub-components that are no longer available.

Additionally, there are ion-chamber-based beam-loss measurements that will enhance the operation of the LANSCE facility, and the $1 \mathrm{~L}$ profile measurement whose present realization is a 2 -axis harp that has several wires

\footnotetext{
*Work supported by US Department of Energy.

"gilpatrick@lanl.gov
}

missing.

While most of these devices have to operate at various physical locations within the LANSCE facility, generally, each of these measurement systems must operate under several operational modes or "flavors".

\section{LANSCE BEAM "FLAVORS"}

To understand the various operational modes or "flavors", one must first understand the present operation of the LANSCE facility. Once extracted from their independent sources, the $750-\mathrm{keV} \mathrm{H}^{+}$and $\mathrm{H}^{-}$beams are combined in their low-energy transports by temporally multiplexing and injecting them into $16.7-\mathrm{MHz}$ and 201.25-MHz bunching cavities. There they are partially bunched in preparation for their injection into the Drift Tube Linac (DTL), where the beams' 201.25-MHz time structures are maintained while they are being accelerated to $100 \mathrm{MeV}$. The $\mathrm{H}^{+}$beam typically has a $30-\mathrm{Hz}$ Repetition Rate (RR) and a 0.625 -ms long macropulse resulting in a Duty Factor (DF) of $1.875 \%$. The $\mathrm{H}^{-}$beam has a more complicated time structure consisting of up to a $40-\mathrm{Hz}$ RR and a $0.625-\mathrm{ms}$ long macropulse. This macropulse is further subdivided or chopped into $\sim 1750$ minipulses and consists of 300-ns-long beam pulses repeated with a RR of $2.8 \mathrm{MHz}$, the $72^{\text {nd }}$ sub-harmonic of the 201.25-MHz bunching time structure. Ultimately, the minipulse length limits the measurement's ability to acquire valid beam-position and -phase information.

As both beams emerge from the DTL, they are magnetically separated. The $\mathrm{H}^{-}$beam's central phase delay is adjusted so that when the two beams are recombined prior to injection into the $805-\mathrm{MHz}$ linac, each beam's central phase matches the proper linac RF bucket. The complicated transport line that accomplishes the adjustment of the $\mathrm{H}^{-}$beam's central phase with minimal disturbance to the beam's transverse characteristics is called the Transition Region (TR). The TR output beams are injected into the $805-\mathrm{MHz}$ side coupled linac that accelerates these beams from 100 to $800 \mathrm{MeV}$. In the past (and future), both beams were (and will be) accelerated to $800 \mathrm{MeV}$ but presently only the $\mathrm{H}^{-}$ beam is injected into the linac. A spur beam line located near the beginning of the TR allows the $\mathrm{H}^{+}$beam to be transported to the Isotope Production Facility (IPF) beam line. Table 1 describes these conditions commonly called beam "flavors" or types of beams and timing structures that are transported through the linac and associated experimental areas, such as the Proton Storage Ring (PSR), the Proton Radiography (pRAD), the Ultra Cold Neutron (UCN), and a future Materials Test Stand (MTS). 
Table 1: Important LANSCE Linac Beam "Flavors"

\begin{tabular}{|c|c|c|c|c|c|c|c|}
\hline \multirow{2}{*}{$\begin{array}{l}\text { Exp. } \\
\text { Area }\end{array}$} & \multirow{2}{*}{$\begin{array}{l}\text { Beam } \\
\text { Type }\end{array}$} & \multicolumn{2}{|c|}{ Minipulse } & \multicolumn{2}{|c|}{ Macropulse } & \multirow{2}{*}{$\frac{\mathbf{I}_{\mathbf{p k}}}{(\mathrm{mA})}$} & \multirow{2}{*}{$\frac{\mathbf{I}_{\text {Avg }}}{(\mu \mathrm{A})}$} \\
\hline & & $\begin{array}{l}\mathrm{DF} \\
(\%)\end{array}$ & $\mathrm{RR}$ & $\begin{array}{l}\mathrm{DF} \\
(\%)\end{array}$ & $\begin{array}{c}\mathrm{RR} \\
(\mathrm{Hz})\end{array}$ & & \\
\hline IP & & & & & & 0 & \\
\hline MTS & & /A & J/A & 3.75 & 60 & 20 & 80 \\
\hline $\mathrm{CN}$ & & 1 & .4 & .8 & 30 & 0 & 60 \\
\hline PSR & & 84 & 2.8 & 2.5 & $<40$ & 10 & 20 \\
\hline $\mathrm{AD}$ & & 34 & $\overline{5.4}$ & 0.3 & 30 & 0.3 & 100 \\
\hline WNR & $\mathrm{H}-$ & 0.008 & 5.4 & 6.25 & $<100$ & $\sim 0.7$ & $\sim 5$ \\
\hline
\end{tabular}

The last row of Table 1 shows a particular beam "flavor" associated with the Weapons Neutron Research (WNR) experimental facility. Presently, each of the beam "flavors" is in its own individual $60-\mathrm{Hz}$ half cycle or 8.3ms period, except for those beams tuned for WNR. Unlike the other beam "flavors," this particular beam occupies the same macropulse period but in opposite 180deg RF-phase buckets as the IPF beam. This $\mathrm{H}^{-}$beam has $\sim 2.5 \mathrm{X}$ the charge in a single RF bucket and can be separated by multiples of $\sim 1.8 \mu$ s, the 360th sub-harmonic of the 201.25-MHz bunching-time structure. Therefore, during nominal production operation, the BPPM signals will be a combination of the two species drifting through each BPPM bore, resulting in some additional measurement complications. Since wire scanners are only used for tune-up purposes and not for production beams, this operational mode for wire scanners is less of a concern.

\section{NEW LINAC BPPM REQUIRED}

The LANSCE $805-\mathrm{MHz}$ linac has a series of legacy beam-position monitors (BPMs) that contain four B-dot loops for beam-position measurements and a cylindrical capacitive pickup, known as the "Delta-T loop." The Delta-T loops provide the 201.25-MHz bunched-beam signals to measure the beam's central phase with respect to a reference and determine the time of flight between accelerating cavities. This phase and time-of-flight information allows the LANSCE accelerator operators and operational scientists to set the accelerating modules' RF-fields phase and amplitude. Unfortunately, the B-dot position measurements do not reliably provide beamposition information.

Since there are no dependable non-interceptive beamposition measurements within this 48-module linac, the linac beam steering is corrected using beam-centroid information from slow wire scanners, and loss monitors. These measurement dependencies cause the linac tuning to be slow and eliminate auto-tuning possibilities. Therefore, new BPPM measurement systems are being developed for the 805-MHz linac.

\section{BPPM Measurement Requirements}

Table 2 shows the BPPM measurement system's overall requirements, including such parameters as beam position, phase, and bunched-beam current [2].
Table 2: Overall BPPM Measurement Requirements

\begin{tabular}{|c|c|}
\hline Parameter & Value \\
\hline Position Repeatability (mm) & 0.1 \\
\hline Position Accuracy (mm) & 1 \\
\hline Beam Position Range ( $\pm \mathrm{mm})$ & 13 \\
\hline Beam Position Response Time ( $\mathrm{ss})$ & 2 to 5 \\
\hline Phase Repeatability (201.25-MHz-degrees) & 0.25 \\
\hline Phase Accuracy (201.25-MHz-degrees) & N/A \\
\hline Phase Range (201.25-MHz-degrees) & $+/-180$ \\
\hline Beam Phase Response Time ( $\mu \mathrm{s})$ & 50 to 100 \\
\hline Current Repeatability (mA) & 0.05 \\
\hline Current Accuracy (mA) & N/A \\
\hline Current Range (mA) & 21 to 0.9 \\
\hline Current Response Time ( $\mu \mathrm{s})$ & 2 to 5 \\
\hline
\end{tabular}

There are two general goals for the placement of these BPPMs, namely to place a subset of BPPMs so that they provide both position and trajectory-angle information, and to place BPPMs in the locations now populated by older and existing Delta-T loops, resulting in 19 locations within the linac.

Operational experience has shown that to minimize the beam losses in the linac, the $\mathrm{H}^{+}$or $\mathrm{H}^{-}$beams are injected into the $805-\mathrm{MHz}$ linac such that established positions are achieved at a number of wire-scanner locations. Once injected, the two beams are not steered throughout the rest of the linac. The proposed steering algorithm for placing the beam on the proper trajectory involves beam-position measurements before and after adjustment of upstream horizontal and vertical steerers. The resulting data are used to determine the relevant transfer-matrix elements between steerers and BPPMs, and subsequently, the proper settings of the steerers.

Through a set of simulations, locations in the linac were determined where BPPMs should be placed for the steering algorithm to work under a wide range of beamoptics conditions, such as for linac quadrupole doublets with settings of between $90 \%$ and $110 \%$ of their historicaverage values and linacs with and without acceleration [3].

\section{BPPM Instrumentation Components}

A different series of RF simulations was performed in order to establish the BPPM mechanical and electrical characteristics [4]. The BPPM measurement systems to be installed will process the beam's fundamental bunching frequency of 201.25 MHz. Since the design's intent is not to detect and measure much higher signal frequencies, the simulations showed that there were two primary designs of interest. One design showed the four electrodes terminated in their characteristic impedance and the other design showed its four electrodes shorted to ground. Both of the designs studied operated equally well at the fundamental bunching frequency in the simulations and in the field. Ultimately, the choice between the two designs was based on reliability and complexity due to the 
fewest number of SMA coaxial feedthroughs used, resulting in the shorted-electrode design being chosen.

A set of mechanical design criteria were developed to simplify overall mechanical requirements [5]. For example a single mechanical design will be used for the BPPMs throughout the $805-\mathrm{MHz}$ linac. Given this single design, all BPPM electrodes will be as long as possible and will have 60-deg subtended angle. A strong electromechanical connection, such as a braze joint, between the feedthrough inner conductor and the electrode will be used. Alignment tooling on the BPPM body will provide a common interface between the BPPM's mapped characterization and its final non-adjustable beam line laser-tracker-measured location.

From previously discussed studies and other input, including a theoretical analysis of beam-position monitors, BPPM signal parameters were estimated. Using these listed parameters, a simulation of In-phase and Quadrature-phase (I/Q) processor channel was performed [6]. This processor channel consisted of a 201.25-MHz band-pass filter, an amplifier, and a 14-bit Analog-to-Digital Converter (ADC). If the ADCs are clocked at a rate which "undersamples" the fundamental bunching period of the four filtered BPPM-electrode signals such that at least one I- and Q-sample is acquired during a single 60 -ns minipulse, both beam magnitude and phase information may be reconstructed from the acquired samples. The resultant studies showed that a BPPM processing-electronics design is feasible using sampled-signal digital processing techniques.

\section{NEW WIRE SCANNERS REQUIRED}

The slow wire-scanner profile measurement is the primary method of measuring the beam transverse distributions throughout the LANSCE facility. From these distributions, the operators and physicists responsible for the machine's tune judge, based on this profile, whether the machines tune is proper. These wire scanner profile measurements are critical to the operation of LANSCE. Unfortunately, most of the existing 122 wire scanners at LANSCE are very old, many of the beam-line devices and electronics are greater than 20 years old. Most of the actuator electronics contain parts that can no longer be acquired and expend a lot of technician labor committed to maintaining this existing set of older equipment.

The goal for the improvements to the wire scanners for the LANSCE-R project from the beam-diagnostics perspective is to update the mechanical, electronic, cabling, and software designs so that the new wirescanner measurement system will be more reliable, and easier and less costly to maintain [7].

Amongst the present wire scanners, there are approximately seven mechanical designs of slow wire actuators with at least four different designs of wire fork arrangements. The actuators use older standard stepper motors, for which there are newer higher-torque models. There are two different electronic designs of the actuator driver electronics and four different controller techniques that create the stepper-motor pulses. There are at least four different designs of the electronics that sense the wire's secondary-electron emission flow as the wire is passed through the beam. There are multiple support electronics and bias power supplies. Finally, the software used to support all of this hardware assumes a constant velocity profile and applies no feedback in a responsive fashion.

The resulting set of profile measurements is difficult to maintain and a major contributor to the beam-diagnostics team's maintenance effort. Fortunately, many of the wire-scanner measurement system components can be replaced by commercial off-the-shelf and readily available components.

\section{ADDITIONAL MEASUREMENT SYSTEM IMPROVEMENTS}

Finally, there are several special systems that are also being considered for replacement. For example, ionization-chamber loss monitors are being considered for installation, for further improvements to the maintenance of the facility.

\section{SUMMARY}

This paper has quickly reviewed the beam-diagnostics instrumentation goals for the upcoming LANSCE-R project. It has provided some limited detail for the proposed improvement of the BPPM measurement systems for the linac. Furthermore, this paper has also described why slow wire-scanner profile measurements are being improved. Finally, this paper has briefly listed additional measurements that are being considered for LANSCE-R, such as ionization-chamber loss monitors.

\section{REFERENCES}

[1] Kevin Jones, "The LANSCE Accelerator System Refurbishment (LANSCE-R)," this conference.

[2] J. D. Gilpatrick, et al., "Proposed Beam Position And Phase Measurements for the LANSCE Linac," DIPAC07, Maestra, Italy, May 2007, http://www.elettra.trieste.it/dipac07/ or http://www.jacow.org/

[3] Barbara Blind, private communication.

[4] Sergey Kurennoy, "Electromagnetic Modeling of Beam Position and Phase Monitors for LANSCE Linac," this conference.

[5] James Francis O'Hara, et al., "LANSCE Prototype Beam Position and Phase Monitor (BPPM) Mechanical Design," this conference.

[6] John Power, private communication.

[7] John Power, et al., "Development of Upgraded Wire Scanner Systems for LANSCE," this conference. 